\title{
MOOC: medición de satisfacción, fidelización, éxito y certificación de la educación digital
}

\section{MOOCs: measuring satisfaction, loyalty, success and certification of digital education}

\author{
Ángel González de la Fuente \\ Telefónica Educación Digital, TED (España) \\ David Carabantes Alarcón \\ Universidad Complutense de Madrid, UCM (España)
}

\section{Resumen}

Los MOOC (Massive Open Online Courses) han evolucionado a un formato reconocido en la educación digital, convirtiéndose en un estándar para el aprendizaje, haciéndolos atractivos para los usuarios, promoviendo su difusión y crecimiento. Se realizaron dos encuestas con 3.025 respuestas desde la plataforma MiríadaX a una población de 48.252 usuarios, participantes que finalizaron y no finalizaron cursos durante el primer semestre de 2016, analizando la satisfacción y fidelización. Se ha demostrado una alta satisfacción en las expectativas del curso (93.9 \%) entre los finalizados que contestaron la encuesta. Con valores muy altos aparece la recomendación del curso (80.3 \% entre finalizados / 75.4 \% entre no finalizados) y el interés en seguir cursando MOOC (94\% finalizados / 90 \% no finalizados). Están satisfechos o muy satisfechos con la plataforma un $98.1 \%$ de finalizados y $93.6 \%$ de no finalizados. Más del $44 \%$ de los no finalizados argumentaron falta de tiempo y el elemento más valorado fue siempre el vídeo. Con los datos de registros en MiríadaX, se evaluó la adopción y éxito del formato MOOC. El número de participantes y cursos se ha incrementado progresivamente en MiríadaX desde 2013, con 2.342.00o inscritos y 460 cursos hasta junio de 2016, el ritmo de crecimiento se ha doblado ese año. Otra encuesta con 980 respuestas muestra que más del 59 \% estaría interesado en obtener certificados oficiales de pago. Los MOOC se valoran como un formato de éxito y son el futuro de la adquisición de conocimiento online.

Palabras clave: MOOC; satisfacción; fidelización; éxito; certificación; educación digital.

\section{Abstract}

The MOOCs (Massive Open Online Courses) have evolved into an educational digital format, becoming a standard, which makes them more attractive to Internet users, promoting its dissemination and growth. Surveys to analyze satisfaction and loyalty were sent to 48.252 users who completed and not completed courses in MiríadaX during the first half of 2016, receiving 3.025 answers. As well, data records from MiríadaX were used for evaluating adoption and success of the MOOC format. Surveys results showed high satisfaction levels 
related to course expectations with a positive $93.9 \%$ among respondents who completed a course, also high levels of course recommendation was registered (among completed: 80.3 \% / not completed: $75.4 \%$ ) and interest in further pursuing MOOCs was high (completed: $94 \%$, not completed: $90 \%)$. Satisfaction with the platform showed a $98.1 \%$ (completed) and $93.6 \%$ (not completed). More than $44 \%$ of not completed course respondents argued lack of time and the most valued element was always the video. The number of participants and courses has progressively increased since 2012 in MiríadaX, with 2.342.00o registered and 460 courses so far in June 2016, doubling the ratio of growth in the last year. Another survey with 980 answers showed that more than $59 \%$ of respondents would be interested in obtaining a paid official certificate from the course. The MOOCs are valued as a successful format and the future of online learning.

Keywords: MOOC; satisfaction; loyalty; success; certification; digital education.

Desde la aparición del curso CCKo8-Connectivism and Connective Knowledge (Fini, 2009), considerado el primero de los MOOC, los cursos en línea, masivos y en abierto han sido una fuente de debate académico desde su aparición ante el gran público en 2012. Como recoge García Aretio (2015), de una primera fase de acogida entusiasta ante los primeros resultados de inscripciones, hasta la decepción sobre sus tasas de finalización (Jordan, 2014), se ha debatido en variables sobre su validez académica y formativa, sobre si son moda o tsunami.

Si tenemos en cuenta el análisis de la gráfica de tendencias de la consultora Gartner desde artículos como el de Zapata-Ros (2013) y Cabero et al. (2014), los MOOC se encontrarían en los primeros pasos de la gráfica, y en publicaciones como las de Poy y Gonzales-Aguilar (2014) junto con la de Cabero (2015), planteaban que los MOOC iniciaban la curva descendente.

En cualquier caso, se puede afirmar que los MOOC siguen manteniendo su potencial intacto: la educación digital. Gracias a ello, un número siempre creciente de usuarios los están utilizando, y así lo demuestra la progresión de usuarios de las plataformas, como se puede consultar en OnlineCourses.com (2016).

Las necesidades de formación electrónica en la sociedad siguen creciendo y los MOOC son la principal puerta de entrada al conocimiento, dado que su estructura sencilla y asequible ha sido acogida por la demanda de formación. Como indican los autores Del Moral y Villalustre (2015), los MOOC disponen además de elementos que permiten desarrollar entornos personales de aprendizaje (PLE).

La concepción de lo que es un MOOC ha variado de forma importante en el tiempo escaso que lleva como oferta en el mercado educativo. Existen distintas formas de clasificar los MOOC, si ya antes de que se implantara de forma regular existía una variedad de formatos como los que recoge Cabero et al. (2014) alineados con distintos planteamientos (cMOOC, xMOOC, etc.), hoy día puede definirse casi exclusivamente con unas reglas bastante definidas que, al menos, en lo descriptivo conforman una estructura que está rápidamente convirtiéndose en algo parecido a un estándar, lo que a su vez ayuda a su generalización, acogida, difusión y éxito. 
En ese sentido, un MOOC es un curso online de varias semanas, entre 4 y 10, con una fecha de inicio y otra de fin, apoyado principalmente por una oferta de vídeos con charlas formativas, material adicional de descarga, referencias en la red, ejercicios y tests auto evaluativos, actividades de evaluación entre pares (peer to peer o P2P), y un esquema de foros y herramientas sociales para la generación de una comunidad alrededor del aprendizaje. El número de horas de estudio no suele superar las $4 / 5$ horas de trabajo semanal y cada semana suele corresponderse con un módulo de formación, estando la mayoría de las veces todos los módulos abiertos desde la fecha de inicio. Distintos autores como Valverde (2014) han indagado en las peculiaridades tan específicas de estos cursos.

La característica principal de este formato es la comunicación en vídeo, que en sí misma también está convirtiéndose en un estándar con similitudes muy marcadas en la mayor parte de los MOOC (Reutemann, 2016), donde el elemento principal es la transmisión de conocimientos mediante una charla de un docente que se dirige a sus estudiantes a través de un plano medio (busto) y que elabora unos planteamientos apoyados muchas veces por diapositivas o refuerzos de mensajes en el plano. Cabe destacar en este formato la creciente importancia de la capacidad de comunicación del docente a través de técnicas como dirigirse directamente a cámara y buscar la complicidad continua de la audiencia, habilidades muy relacionadas con las artes antiguas de la oratoria y la retórica. La credibilidad de los cursos en muchas ocasiones se dirime por esa capacidad, y no sólo por los contenidos y el diseño instruccional, lo que es muy importante que los creadores de nuevos MOOC tengan en cuenta. Incluso se da el hecho que en ocasiones se sustituye a los docentes por actores que, a partir de un guion, son los encargados de enfrentarse a la cámara.

El formato MOOC, así definido, ha superado los planteamientos de sus propias siglas, por lo que también ha sido criticado, ya que se está utilizando en la actualidad, y cada vez más, para todo tipo de formación mediada por Internet, donde las siglas de Massive (masivo) y Open (abierto) se aplican por igual en cursos que tienen el objetivo de abastecer a pocos usuarios en un formato exclusivo o de pago. De sus altas ambiciones instruccionales de inicio se ha quedado en lo más básico, en ser capaz de motivar a los estudiantes creando un espacio de aprendizaje que combinan unas obligaciones junto a una comunidad, que asemejan las condiciones más simples de la educación tradicional, pero en el terreno virtual.

De entre todas las plataformas, destaca en el ámbito iberoamericano MiríadaX, y como recogen Capdevila y Aranzadi (2014) dicha plataforma está provocando una serie de cambios en el sistema universitario.

En este trabajo se intenta comprobar cuál es el grado de satisfacción con el formato MOOC, el índice de fidelización, -revisando las tasas de crecimiento de inscripciones a los cursos para ver si su adopción ha sido un éxito-, cuál es el principal impedimento para finalizar los cursos y si los alumnos están empezando a demandar un paso más en la formación: las certificaciones oficiales. 


\section{METODOLOGÍA}

Al no existir una escala validada para la medición de los aspectos fundamentales de este estudio, se confeccionó una encuesta de diez preguntas a ser respondida desde enero a mayo de 2016 por usuarios que finalizaron al menos un MOOC de la plataforma MiríadaX.

Las preguntas fueron: 1) Por favor, indícanos en qué rango de edad te sitúas, 2) ¿Cuál es tu nivel de estudios?, 3) Género, 4) Una vez que has realizado el curso, ¿qué nos puedes decir sobre las expectativas que tenías?, 5) ¿Recomendarías este curso? (teniendo en cuenta que daremos un 1 si no lo recomendaríamos nada y un 10 si lo recomendaríamos mucho), 6) Por favor, valora la implicación del equipo docente en el curso, 7) Por favor, marca tu grado de satisfacción con los contenidos del curso, 8) Indica tu grado de interés en seguir realizando cursos MOOC (teniendo en cuenta que 1 es ningún interés y 10 mucho interés), 9) ¿Cuál es tu grado de satisfacción con la plataforma MiríadaX? y 10) ¿Lo compartirías con tus contactos?

En las mismas fechas también se difundió una encuesta de 11 preguntas a ser respondida por usuarios que no finalizaron un MOOC entre participantes de cursos registrados en la plataforma MiríadaX, utilizando las mismas preguntas que en el cuestionario para los que finalizaron los cursos, excepto la pregunta número 4 que fue sustituida por estas otras dos: ¿Cuál fue tu grado de avance en el curso? y ¿Cuál fue el motivo para no finalizar el curso?

Las encuestas se remitieron a 48.252 participantes de 10 cursos:

- Seguridad Nutricional: 3.290 participantes.

- Potencia tu mente ( $5^{\mathrm{a}}$ edición): 11.918 participantes.

- Habilidades y competencias a través del coaching personal (5a edición): 13.167 participantes.

- Paz y Noviolencia ( $3^{\text {a }}$ edición): 788 participantes.

- El abordaje multidisciplinar de las demencias: 4.254 participantes.

- Ética en la investigación universitaria: 1.263 participantes.

- Desentrañar el firmamento-Introducción a la Astronomía: 7.171 participantes.

- Seguridad del Paciente ( $5^{\mathrm{a}}$ edición): 2.040 participantes.

- Estética y Teoría del Arte en el siglo XVIII: 2.654 participantes.

- Nuevos escenarios de aprendizaje digital: 1.707 participantes.

Para calcular los avances en la plataforma se han utilizado los datos de registros en cursos de MiríadaX de los últimos 3 años: 2014, 2015 y 2016.

Por último, se elaboró otra encuesta de febrero a junio de 2016 entre usuarios de los últimos doce meses de la plataforma MiríadaX, que hubieran finalizado o no el 
curso, con las siguientes 8 preguntas: 1) ¿Has comprado el certificado de superación de alguno de nuestros cursos?, 2) En caso de haber respondido afirmativamente la pregunta anterior, valora tu satisfacción con la compra del certificado de superación, 3) ¿Estarías interesado en completar tu próximo curso y obtener un certificado oficial acreditado por la Universidad por un total de $\$ 200$ (incluyendo matrícula y tasas universitarias)?, 4) ¿Cuál es la probabilidad de que decidieras invertir \$200 en la matrícula y las tasas de acreditación oficial de un curso? siendo 1 muy baja probabilidad y 10 muy alta, 5) Por favor, indícanos en qué rango de edad te sitúas, 6) ¿Cuál es tu nivel de estudios?, 7) ¿Desde dónde realizas los cursos? y 8) Género.

\section{RESULTADOS}

Para comprobar la adopción del formato se midió el índice de satisfacción de los usuarios de los MOOC, divididos entre aquellos que hubieran finalizado los cursos y los que no. Más de tres mil participantes (3.025) contestaron estas dos encuestas, 1.828 finalizados y 1.197 no finalizados.

Si empezamos por los finalizados, de las 1.819 respuestas que contestaron la pregunta sobre las expectativas, sólo un $6 \%$ manifestó que el curso realizado no había satisfecho sus perspectivas y del resto destaca que se cumplieron satisfactoriamente las expectativas que se tenían. (Figura 1).

Figura 1. Expectativas una vez realizado el curso

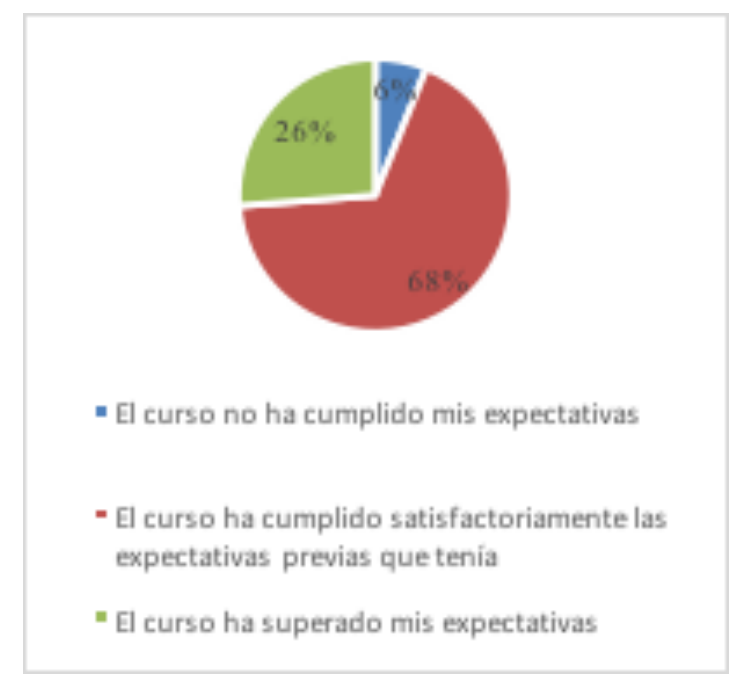

Sobre si recomendarían el curso recién finalizado, y con una valoración de 1 a 10, siendo 10 que totalmente lo harían, los resultados de las 1.819 respuestas 
en dicha pregunta, mostraron que los valores entre 8 y 10 (alta posibilidad real de recomendación) se encuentra más del 80 \% de los encuestados que finalizaron el curso. Si hacemos el mismo análisis entre las respuestas de los 1.115 participantes que no finalizaron los cursos y contestaron a esa misma pregunta, un 7,5\% recomendarían el curso recién finalizado, y con valores entre 8 y 10 se encuentra el $75 \%$ de los encuestados, con un 7,5\% que manifiesta que no avanzó lo suficiente para hacer una valoración. (Figura 2).

Figura 2. ¿Recomendarías este curso?

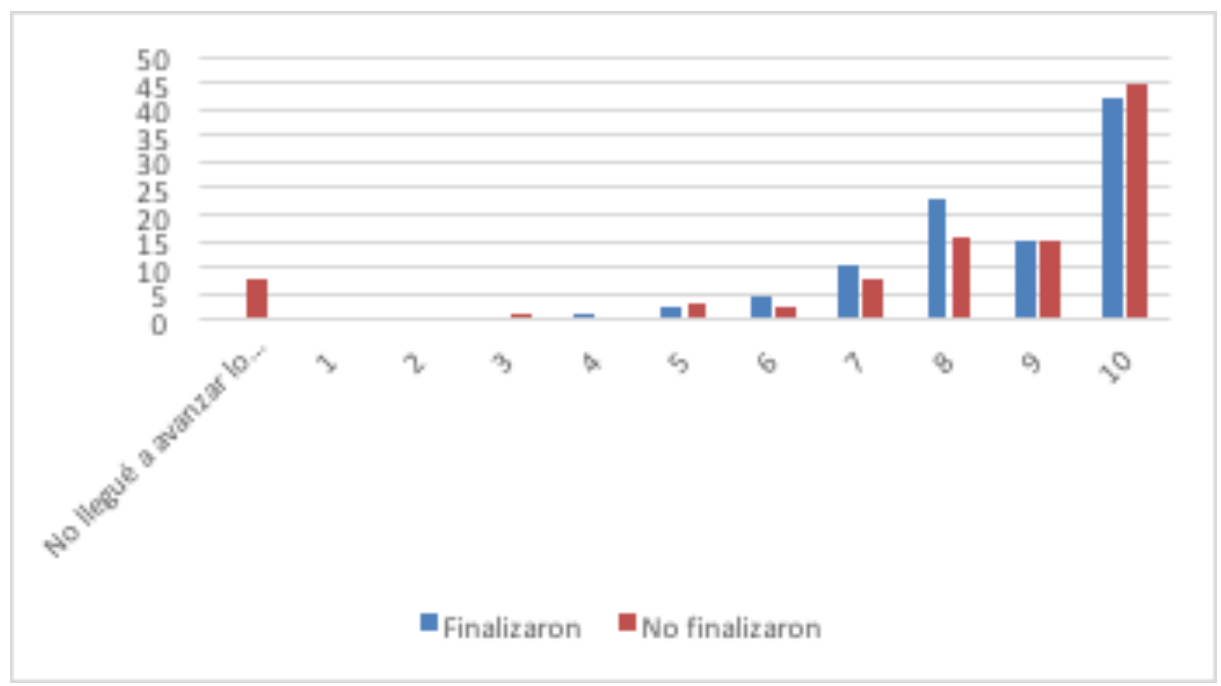

$\mathrm{Si}$ valoramos con las 1.791 respuestas los diferentes elementos del curso, comprobamos que el más satisfactorio son los vídeos, seguido por los ejercicios y tests auto evaluativos, el primero con un $96 \%$ de respuestas entre satisfactorio y muy satisfactorio, y el segundo con un $94 \%$. Ante la evaluación por participantes que no han finalizado el curso, se puede volver a comprobar con las 1.066 respuestas, la prevalencia de los vídeos didácticos y de los ejercicios y de tests auto evaluativos. En ambos casos el elemento menos valorado fue la participación en los foros. (Tabla 1). 
Tabla 1. Grado de satisfacción con los contenidos del curso finalizado

\begin{tabular}{|c|c|c|c|c|c|c|c|c|c|c|}
\hline & \multicolumn{2}{|c|}{$\begin{array}{c}\text { Altamente } \\
\text { satisfactorio }\end{array}$} & \multicolumn{2}{|c|}{ Satisfactorio } & \multicolumn{2}{|c|}{$\begin{array}{c}\text { Me ha dejado } \\
\text { indiferente }\end{array}$} & \multicolumn{2}{|c|}{$\begin{array}{l}\text { Poco o nada } \\
\text { satisfactorio }\end{array}$} & \multicolumn{2}{|c|}{$\mathrm{N} / \mathrm{C}$} \\
\hline & 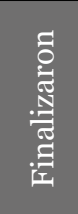 &  & 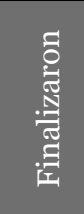 & 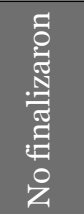 & 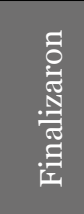 & 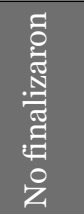 & 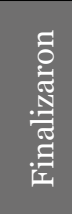 & 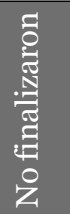 & 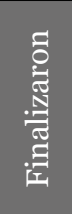 &  \\
\hline $\begin{array}{l}\text { Visualización } \\
\text { de los vídeos } \\
\text { didácticos }\end{array}$ & 973 & 528 & 760 & 396 & 35 & 33 & 10 & 19 & 2 & 90 \\
\hline $\begin{array}{l}\text { Lectura de los } \\
\text { contenidos } \\
\text { adicionales o } \\
\text { descargables }\end{array}$ & 785 & 470 & 850 & 427 & 82 & 42 & 48 & 24 & 23 & 102 \\
\hline $\begin{array}{l}\text { Participación } \\
\text { en las } \\
\text { actividades } \\
\mathrm{P} 2 \mathrm{P}\end{array}$ & 400 & 197 & 751 & 414 & 204 & 111 & 48 & 70 & 363 & 271 \\
\hline $\begin{array}{l}\text { Participación } \\
\text { en los foros }\end{array}$ & 259 & 150 & 743 & 352 & 335 & 187 & 61 & 62 & $35^{8}$ & 306 \\
\hline $\begin{array}{l}\text { Realización } \\
\text { de } \\
\text { cuestionarios } \\
\text { y actividades } \\
\text { de tipo test }\end{array}$ & 801 & 401 & 875 & 436 & 60 & 48 & 33 & 42 & 13 & 135 \\
\hline
\end{tabular}

Una vez estudiada la satisfacción media con los MOOC, se intenta evaluar si el formato fideliza a los usuarios, midiendo si recomendarían su uso, no tanto del curso que acaban de realizar, más bien por el formato MOOC.

Entre los participantes que finalizaron los cursos, las 1.746 respuestas a la pregunta de si volverían a hacer un MOOC, con una valoración entre 1 y 10 (siendo 10 estar completamente seguros de que repetirían), más del $93 \%$ se situó en una valoración entre 8 y 10. Además, el valor 10 tuvo un 62 \% de las respuestas, o lo que es lo mismo, 6 de cada 10 de los alumnos que finalizaron un curso manifiestan una completa seguridad en seguir realizando MOOC. Con las 1.036 respuestas de los que no finalizaron cursos, también se obtuvieron resultados similares con un $90 \%$ con una valoración muy positiva (entre 8 y 10); el 10, la opción de estar completamente seguro de repetir, tuvo un $64 \%$ de respuestas. (Figura 3). 
Figura 3. Grado de interés en seguir realizando cursos MOOC

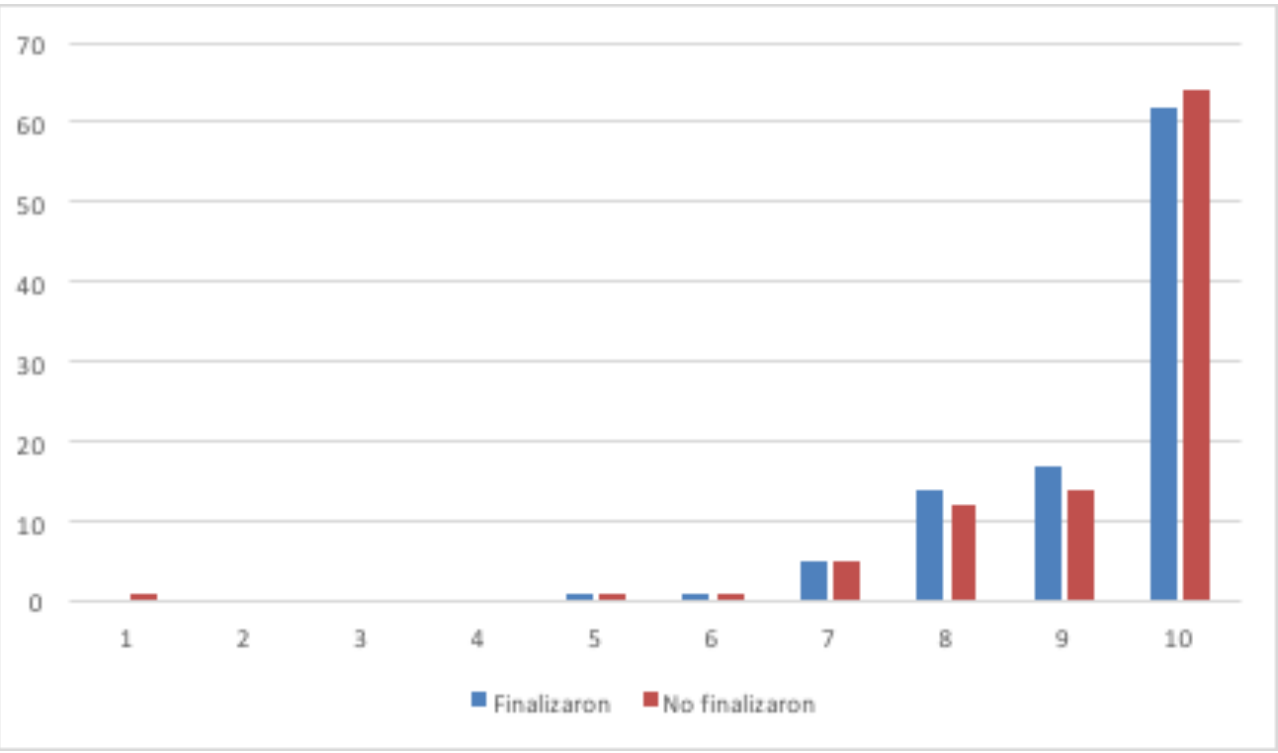

Ante la pregunta del grado de satisfacción con la plataforma MiríadaX con las 1.746 respuestas de los que finalizaron el curso, tan solo un o,8 \% manifestó estar poco o nada satisfecho con la misma. Las 1.036 respuestas de los que no acabaron es indicativo de alta satisfacción; la poca o ninguna satisfacción únicamente alcanzó el $2,4 \%$. (Tabla 2).

Tabla 2. Grado de satisfacción con la plataforma MiríadaX

\begin{tabular}{|l|c|c|c|c|c|c|c|c|}
\hline & \multicolumn{2}{|c|}{$\begin{array}{c}\text { Altamente } \\
\text { satisfactorio }\end{array}$} & \multicolumn{2}{c|}{ Satisfactorio } & \multicolumn{2}{c|}{$\begin{array}{c}\text { Me ha dejado } \\
\text { indiferente }\end{array}$} & \multicolumn{2}{c|}{$\begin{array}{c}\text { Poco o nada } \\
\text { satisfactorio }\end{array}$} \\
\hline Finalizaron & 986 & $56,47 \%$ & 727 & $41,64 \%$ & 19 & $1,09 \%$ & 14 & $0,80 \%$ \\
\hline No finalizaron & 587 & $56,66 \%$ & 383 & $36,97 \%$ & 41 & $3,96 \%$ & 25 & $2,41 \%$ \\
\hline
\end{tabular}

En cuanto a las razones para no acabar el MOOC, de los 1.115 participantes que no finalizaron el curso, más de un $40 \%$ se justifica en que no tuvo tiempo suficiente para acabarlo. (Figura 4). 
Figura 4. Motivo para no finalizar el curso

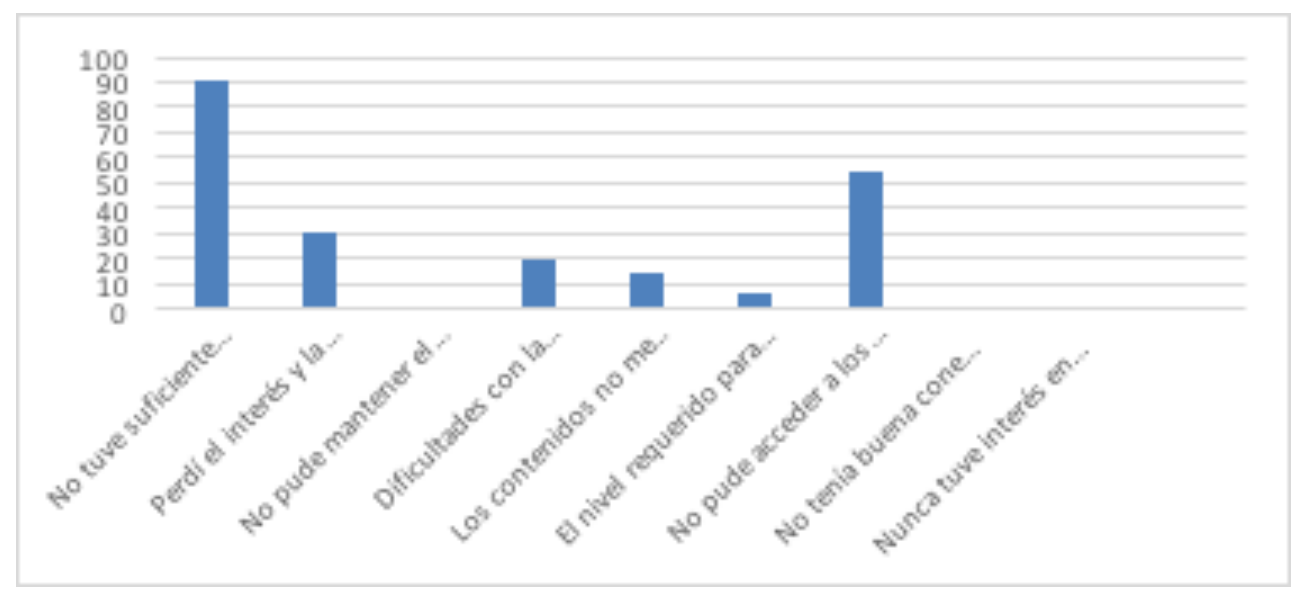

Para completar esta información, gracias al acceso a los datos de registros en los cursos de la plataforma de MiríadaX, se ha podido comprobar que más de la mitad de los alumnos que se registraron en un curso se han inscrito en al menos dos cursos más desde 2014. En junio de 2016 había más de 2.300.00o de registros en los cursos de la plataforma.

Contamos con el acceso a los datos generales de registros en cursos de la plataforma MiríadaX, mostrando un crecimiento en número de alumnos con 1.055.00o alumnos registrados en 2014, 1.726.000 en 2015 y a principios de junio de 2016 ya se habían superado los 2.330.000 inscritos.

El ritmo en las 22 primeras semanas de 2016 significó registrar más de 25.000 alumnos a la semana a los MOOC de esta plataforma. En cuanto a los números de nuevos MOOC, MiríadaX cerró el año 2014 con 219 cursos, 2015 con 371 y en junio de 2016 ya había 461. La media de alumnos por curso en MiríadaX supera los 5.00o alumnos.

Con el fin de estudiar el tema de la certificación oficial, el 10 de febrero de 2016 se difundió una nueva encuesta entre alumnos registrados en cursos de MiríadaX en los doce meses anteriores, con 980 respuestas, en las que preguntaban si el usuario estaría interesado por una certificación oficial previo pago de una matrícula y tasas por un precio de hasta 200 dólares. Las respuestas revelaron que un $31 \%$ de los alumnos contestaban afirmativamente a la pregunta de estar interesados, y que un 29 \% más lo harían dependiendo de la universidad que realice la acreditación. (Figura 5). 
Figura 5. Interés en obtener un certificado oficial acreditado por la Universidad por \$200



- Si - No " Depende de la Universidad que lo acredite

Los encuestados, ante una valoración de 1 a 10 para manifestar su interés por las certificaciones oficiales de pago, muestran en más de un $30 \%$ un alto interés por las mismas (8-10) y un $15 \%$ demuestran un interés máximo (10). (Tabla 3)

Tabla 3. Probabilidad de invertir \$200 para obtener un certificado oficial

\begin{tabular}{|c|c|c|c|c|c|c|c|c|c|}
\hline 1 & 2 & 3 & 4 & 5 & 6 & 7 & 8 & 9 & 10 \\
\hline $13,47 \%$ & $5,71 \%$ & $9,59 \%$ & $7,65 \%$ & $10,10 \%$ & $11,63 \%$ & $10,20 \%$ & $10,61 \%$ & $5,31 \%$ & $15,71 \%$ \\
\hline
\end{tabular}

\section{DISCUSIÓN}

El análisis de la literatura existente permite afirmar que el formato MOOC, con sus características y peculiaridades, está sirviendo para crear un estándar en la educación digital que, a su vez, está colaborando en su desarrollo, porque permite su difusión y conocimiento, su estandarización, como apunta Méndez (2013). Aun así, hay que recordar que, como afirman Torres y Gago (2014) o Cooperman (2014), habría que llegar al mayor número de capas de la sociedad y existen usuarios de Internet que todavía desconocen lo que es un MOOC. Es destacable que desde la página web de la consultora Gartner (2015) aparece MOOC-Enabling Technologies en el inicio de la meseta de la productividad, y en el comienzo de la curva de innovación en la edición del año siguiente (Gartner, 2016) bajo el concepto MOOC Platforms. Estamos sólo en el inicio de una verdadera expansión del modelo, a pesar de ser ya realmente masivo. 
Previamente a la aparición del formato MOOC existían múltiples versiones de cursos online, sin haber sido capaz de desarrollar el potencial de transmisión de conocimientos de forma masiva. Si bien el tópico de que todo el conocimiento está en Internet, se necesita criterio y un formato accesible y amigable como recoge Rodríguez-Ascaso y Boticario (2015).

Por otra parte, Drachsler y Kalz (2016) aplican la analítica de aprendizaje y MOOC y proponen la estandarización del diseño instruccional y de la evaluación como líneas de trabajo en las que hay que profundizar. Un diseño eficaz, como recoge Aguaded y Medina-Salguero (2015), permite mejorar la calidad de los MOOC, y hay propuestas de evaluación de la calidad (Martín et al., 2013, Mengual-Andrés et al., 2015), y existen trabajos como el realizado por Baldomero (2015) que demostraron que los MOOC analizados cumplían los valores de calidad en las tres dimensiones en las que se compone la herramienta utilizada.

Los resultados de las encuestas del presente estudio muestran el altísimo grado de satisfacción de los usuarios que no han finalizado los cursos, en niveles similares a los que sí los han terminado, además de una gran fidelización al formato, que se puede considerar entusiasta. Con otros servicios exitosos de Internet y de la revolución digital se encuentran paralelismos en uso y satisfacción. Aquí el hallazgo ha sido un formato que permite la transmisión de conocimientos. El estándar actual de MOOC establece como una de sus características principales una fecha de inicio y una de fin, aspectos que en un primer momento parece ir en contra de la accesibilidad a la formación y al seguimiento del curso.

Incluso se produce una paradoja muy interesante en cuanto a su uso. Ya hemos reseñado que una de las claves de la adopción de este estándar de formación online se debe a las similitudes virtuales que establece con la formación presencial: de forma muy general, las obligaciones relacionadas con el seguimiento de un curso y la apariencia de una comunidad. Pues bien, una de las obligaciones mejor aceptadas es precisamente la limitación en el tiempo de realización del curso.

De hecho, una de las características habituales de los cursos formativos online previa a la aparición y adopción de este formato MOOC era una continua disponibilidad de la formación, esperando hacerlo más atractivos precisamente a través de una accesibilidad ilimitada, que era posible gracias a las posibilidades tecnológicas. Los resultados son que, muy al contrario de lo que se pretendía, el acceso ilimitado, junto a un bajo compromiso inherente a esa oferta, hace de esos cursos que sean menos motivadores en su inscripción y realización por parte de los alumnos. Lo cual, ciertamente, es una paradoja, porque, como hemos visto en los resultados de las encuestas, es el principal elemento que impide la finalización de los mismos, aunque hay autores que establecen hasta cuatro tipos diferente de alumnos de MOOC, entre los que también destacan aquellos que, por razones de exploración/ curiosidad, o por intereses muy específicos, sólo están interesados en una pequeña parte de la formación ofertada. 
Hay que incidir en el compromiso importante que supone para un usuario apuntarse a un MOOC: le va a exigir entre 3 y 5 horas de trabajo a la semana, lo que no es poco en el mundo virtual en el que el compromiso de los usuarios con un servicio o plataforma se mide en minutos diarios. El compromiso para dedicar esas horas va directamente relacionado con la motivación del alumno por completar todas las tareas, incluso más allá de lo que es el simple acceso del conocimiento que no siempre depende, o va relacionado de forma unívoca, con la realización de todos y cada uno de los posibles ejercicios. Dicho con otras palabras, el alumno puede haberse motivado, y por tanto sacar el tiempo necesario para ello, para ver todos y cada uno de los vídeos formativos, incluso varias veces, pero puede que no encuentre el tiempo necesario para hacer todas las actividades que, por la razón que sea, no le parecen tan interesantes para su formación. Por supuesto, esto no quiere decir que los estudiantes no le vean utilidad al MOOC que han hecho y no completado, como hemos visto en la satisfacción expresada.

Lo que sí parece claro es que los alumnos, a pesar de la paradoja del tiempo/ accesibilidad para realizar los cursos, no consideran este hecho de forma negativa para valorar los MOOC. Precisamente esa, entre otras limitaciones, son los que lo hacen atractivos para la mayoría de ellos y forma parte de ese formato estándar que se está imponiendo. Tampoco parece un impedimento para que cada alumno le saque el provecho que estaba buscando.

En MiríadaX, como en muchas otras plataformas, los alumnos pueden inscribirse en los cursos cerrados bajo el modelo de consulta a los contenidos. Pueden tener acceso a los vídeos y a los materiales adicionales, pero no hay fechas de inicio ni de fin, ni actividades auto evaluativas ni comunidad. Según los datos de la plataforma, sólo uno de cada veinte alumnos opta por esa opción, siendo siempre preferente la de inscribirse a un curso con fecha de inicio cercana o próxima o recién abierto. Es la principal paradoja de la formación online: las obligaciones temporales son más importantes para motivar que para completar la formación. El tiempo, su uso, disfrute y gestión, es cada vez más la moneda de cambio principal de la economía de la revolución digital, y en el caso de la formación, su gran paradoja ha sido descubierta gracias al formato MOOC.

Es importante reseñar que, si bien una de las críticas más frecuentes al formato MOOC provenga de las tasas de finalización, no por ello supone que no sea de gran utilidad para todos los usuarios. Todo lo contrario, las encuestas muestran que les ha sido igualmente útil en aquello que buscaban, que mayoritariamente recomendarían el curso y que parece claro y que volverán para hacer más. Hay un dato especialmente relevante entre los registros de cursos en la plataforma MiríadaX que indica que, de hecho, es mucho más fácil conseguir que un alumno que ya se haya apuntado a un MOOC, comience otro. Y que aquel que se apunta por primera vez es muy probable que repita.

Los resultados de las encuestas ponen de manifiesto la importancia del vídeo en la comunidad de aprendizaje, como se recoge en estudios sobre estandarización 
del vídeo (Reutemann, 2016). Además, este formato mantiene sus propias normas, busca crear una sensación aparente de inter personalidad, de relación casi íntima entre docente y usuario (típica de la forma de consumo individual de un MOOC) en donde el docente se refiere en exclusiva al estudiante. Esa capacidad de conexión directa con la audiencia puede ser una de las claves de la adopción por los usuarios del formato MOOC. Así lo reconocen los estudiantes marcando este elemento como el más valorado en los cursos y podríamos afirmar que la capacidad de conexión del docente a través de esos vídeos va a ser, junto a la materia y conocimiento de la misma, el principal factor de valoración de calidad entre los usuarios.

En relación a los elementos del curso, existen estudios como el de Alario-Hoyos et al. (2016) que recogen el análisis de las herramientas sociales (Facebook, Twitter, Q\&A, Forum y MentorMob) para mejorar la calidad de un MOOC. El trabajo de Barak et al. (2016) muestra la relación positiva entre motivación y el número de mensajes enviados a los foros. Según Iyer y Katona (2015), el denominado principio de desigualdad participativa (90-9-1) se puede aplicar a redes sociales como Twitter, en el que el $10 \%$ de los usuarios crean más del $90 \%$ del contenido, mientras que el resto son mayoritariamente simples espectadores o lectores. Como indican Barba et al. (2016), se pueden utilizar los resultados de la investigación sobre la influencia en el rendimiento de la participación y la motivación intrínseca para adaptar el diseño e impartición de MOOC. Trabajos como los de Kop (2011a, 2011b) plantean cuestiones sobre los niveles de autonomía de los participantes de los MOOC y otros se centran, como los realizados por Clarà y Barberà (2013), en las características de los cursos basados en comunidades colaborativas de aprendizaje o cMOOC. En cualquier caso, todo esto parece superado por la adopción entusiasta del formato y su capacidad de fidelización.

El otro elemento que siempre ha sido planteado como fundamental en los MOOC es la parte social y colaborativa. Sin embargo, está muy lejos de los altos ratios de valoración del vídeo. Si bien entendemos que a los usuarios les gusta y les motiva formar parte de una comunidad, no siempre la utilizan y no más de un $10 \%$ de los usuarios hacen comentarios en los foros. Este dato es consistente con los de servicios de redes sociales.

Además, la participación en los foros no siempre está enfocada a los comentarios sobre la materia o el motivo de aprendizaje, y tampoco, al igual que en las redes sociales, el tono de los comentarios es similar, observándose en algunos casos comportamientos parecidos a los más negativos que se pueden encontrar en las redes sociales, lo que no favorece que sea el principal elemento a valorar. Dicho esto, sigue siendo una parte fundamental e insustituible del formato.

Los datos presentados de MiríadaX y la valoración de la información que proporciona de forma constante alguna web de seguimiento de este mercado, como el portal Class Central (2015a) con 35 millones de usuarios y un crecimiento que prácticamente dobló el número total de usuarios a los de los tres años anteriores. También recoge que el número mundial de cursos MOOC disponibles creció en 
2015 en un $75 \%$ hasta llegar a los 4.200. Siendo esta una cantidad considerable, sin embargo, es un número muy modesto teniendo en cuenta el total de usuarios, lo que establece una media de 7.000 alumnos por curso. No es ningún secreto entender que buena parte del crecimiento de registro en MOOC aún sigue en paralelo a la oferta de cursos, y que estos, a pesar del crecimiento de la oferta, aún está por detrás de la demanda y de las necesidades de formación. El número total de cursos disponibles se incrementará en un porcentaje de al menos el $50 \%$ o superior, mientras que el número de alumnos registrados doblará su crecimiento respeto a 2015, lo que significa que, a nivel mundial, se superarán con facilidad los 50 millones de alumnos registrados en los cursos, siendo bastante probable que se alcancen los 60 millones, de los cuales unos 3 millones serán alumnos de MiríadaX.

En cuanto al interés de la certificación, autores como Vázquez-Cano y López (2015) afirman que los MOOC no tendrían como objetivo prioritario una acreditación o certificación, mientras que el modelo de negocio ha sido analizado por autores como Dellarocas y Van Alstyne (2013) y aunque es necesario tiempo para determinar lo que ha funcionado, las certificaciones tienen mucho que ver.

El portal Class Central (2015b) alertaba de que terminaba la era MOOC de las certificaciones gratuitas. No solo eso, empieza a acabar la expectativa de que la certificación que se pueda adquirir de un MOOC no vaya más allá del reconocimiento que la plataforma proveedora pueda proporcionar. Si volvemos a la metáfora de la escuela como estructura inspiradora y motivadora del formato MOOC, con sus obligaciones y su comunidad, estamos llegando a la era en la que se empieza a esperar, y pronto se exigirá, que si se completa la formación, se requiere una certificación oficial de las instituciones educativas por el compromiso de tiempo y esfuerzo que conlleva obtenerlo. Los usuarios de MOOC están encantados con el formato y seguramente parte de ello también tenga que ver con la apertura y la accesibilidad (entendida ésta como la capacidad de tener acceso al conocimiento de la educación superior), pero también entienden que la acreditación de ese conocimiento es relevante, y por ello están dispuestos a pagar, siempre que dicha acreditación sea oficial esté reconocida por la institución educativa.

De esta manera se comprende que la diferencia respecto a las certificaciones oficiales es aún más reveladora del interés real delos estudiantes por las acreditaciones que den sentido y reconozcan su esfuerzo y compromiso. Los datos de las encuestas y la tendencia general reflejada en las principales plataformas auguran que este será buena parte del camino que tomen los MOOC en el futuro.

El "valor académico", al que se refieren Capdevila y Aranzadi (2014) con las certificaciones emitidas por las universidades de los MOOC realizados desde la plataforma y que fuesen reconocidos por el Espacio Iberoamericano de Conocimiento, pueden asegurar el futuro de este formato en la educación, y al igual que los entornos virtuales de enseñanza/aprendizaje permitieron el desarrollo de innovación en docencia (Carabantes et al. 2005), los MOOC están produciendo una 
serie de cambios que se están aplicando en la docencia tradicional como las aulas inversas. Parece que justamente ese es el camino que se está tomando.

Sería conveniente que otras plataformas de cursos MOOC hicieran públicos sus datos de uso de los participantes y de las encuestas de opinión que lleven a cabo, e incluso construir una escala validada que permitiera comparar los resultados iniciales aquí expuestos y disponer de un mayor número de artículos científicos para argumentar y contrastar las ideas expuestas.

\section{CONCLUSIONES}

Es indudable la creciente influencia e importancia de los MOOC en el ámbito educativo superior y su interés entre los usuarios. Nos encontramos en el inicio de la influencia de estos cursos online en la sociedad en su conjunto. En la situación actual es muy probable que las necesidades de formación presentes y futuras paralelas al advenimiento de la era digital terminen cumplimentándose a través de inscripciones en MOOC y puede que, en algún momento, el número de alumnos registrados en cursos MOOC sea mayor que el de alumnos registrados en cursos universitarios.

Los índices de satisfacción y fidelización con la recomendación de los usuarios de los MOOC, tanto si finalizaron o no, demuestran la adopción y triunfo del formato, que ha superado las barreras de su propia definición, principalmente en lo de abierto, y se encamina a ser el instrumento que defina la formación a través de Internet en los próximos años, lo que -per se-, es un hallazgo enorme. El gran avance que ha generado el formato MOOC: ser capaz de ordenar y hacer posible la formación sin límites y actualización de conocimientos, sucesos que, por su parte, son típicos de la sociedad de la información y de la revolución digital.

La valoración de los elementos del curso, tanto finalizados como no finalizados, muestran el valor de la comunicación en vídeo como elemento singular más destacado del formato, resaltando los ejercicios y tests auto evaluativos más valorados y se debería potenciar más el uso de los foros de debate. La paradoja del tiempo es fundamental para la finalización de los MOOC y justifica el pasado, presente y futuro de este tipo de cursos.

Los datos de registros en plataformas de MOOC muestran un aumento del número de registros, ritmo de crecimiento y número creciente de alumnos por curso. Todo apunta a que tanto MiríadaX como otras plataformas crecerán aún más deprisa que lo hicieron en el resto de ejercicios desde su aparición, logrando aumentar su presencia en el terreno educativo.

Las certificaciones oficiales pueden ser la principal tendencia a futuro de los MOOC, que además tiene una relación directa sobre la motivación, la disponibilidad y la finalización de MOOC. Hay pocos elementos intrínsecos a la formación que impulsen más la finalización de los cursos, que son utilizados e invertidos en tiempo por parte del estudiante exclusivamente hasta donde le llevan sus intereses formativos. 


\section{REFERENCIAS BIBLIOGRÁFICAS}

Aguaded, I., y Medina-Salguero, R. (2015). Criterios de calidad para la valoración y gestión de MOOC. RIED. Revista Iberoamericana de Educación a Distancia, 18(2), 119-143. doi: 10.5944/ ried.18.2.13579

Alario-Hoyos, C., Munoz-Merino, P. J., Perez-Sanagustin, M., Delgado Kloos, C., y Parada G. H. A. (2016). Who are the top contributors in a MOOC? Relating participants' performance and contributions. Journal of Computer Assisted Learning, 32(3), 232-243. doi: 10.1111/jcal.12127

Baldomero Ramírez-Fernández, M. (2015). La valoración de MOOC: Una perspectiva de calidad. RIED. Revista Iberoamericana de Educación a Distancia, 18(2), 171-195. doi: 10.5944/ried.18.2.13777

Barak, M., Watted, A., y Haick, H. (2016). Motivation to learn in massive open online courses: Examining aspects of language and social engagement. Computers \& Education 94, 49-60. doi: 10.1016/j. compedu.2015.11.010

Barba, P. G. de, Kennedy, G. E., y Ainley, M. D. (2016). The role of students' motivation and participation in predicting performance in a MOOC. Journal of Computer Assisted Learning 32(3), 218231. doi: $10.1111 /$ jcal.12130

Cabero Almenara, J., Llorente Cejudo, M. C., y Vázquez Martínez, A. I. (2014). Las tipologías de MOOC: su diseño e implicaciones educativas. Profesorado: Revista de curriculum y formación del profesorado, 18(1), 13-26.

Cabero Almenara, J. (2015). Visiones educativas sobre los MOOC. RIED. Revista Iberoamericana de Educación a Distancia, 18(2), 39-6o. doi: 10.5944/ ried.18.2.13718

Capdevila Pagès, R., y Aranzadi Elejabeitia, P. (2014). Los Cursos Online Masivos y Abiertos: ¿oportunidad o amenaza para las universidades iberoamericanas? RIED. Revista Iberoamericana de Educación a Distancia, 17(1), 69-82. doi: 10.5944/ ried.17.1.11574

Carabantes Alarcón, D., Alves, J., y Carrasco Pradas, A. (2005). La innovación a través de entornos virtuales de enseñanza y aprendizaje. RIED: Revista Iberoamericana de Educación a Distancia, 8(1-2), 105-126.

Clarà, M., y Barberà, E. (2013). Learning online: massive open online courses (MOOCs), connectivism, and cultural psychology. Distance Education, 34(1), 129-136.

Class Central. By The Numbers: MOOCS in 2015. December 21, 2015. Class Central. Recuperado de https://www.classcentral.com/report/moocs-2015-stats/

Class Central. MOOC Trends in 2015. The Death of Free Certificates. December 14, 2015. Class Central. Recuperado de

https://www.class-central.com/report/ death-of-free-certificates/

Cooperman, L. (2014). From elite to mass to universal higher education: from distance to open education. RIED. Revista Iberoamericana de Educación a Distancia, 17(1), 111-130, doi: 10.5944/ ried.17.1.11576

Daniel, J., Vázquez Cano, E., y Gisbert, M. (2015). El futuro de los MOOC: ¿aprendizaje adaptativo o modelo de negocio? RUSC. Universities and Knowledge Society Journal, 12(1), 64-74. doi: 10.7238/rusc.v12i1.2475

Del Moral Pérez, E., y Villalustre Martínez, L. (2015). MOOC: ecosistemas digitales para la construcción de PLE en la Educación Superior. RIED. Revista Iberoamericana de Educación a Distancia, 18(2), 87-117. doi: 10.5944/ried.18.2.13353

Dellarocas, C., y Van Alstyne, M. (2013). Money models for MOOCs. Considering new business models for massive 
open online courses. Communications of the ACM, 56(8), 25-28. doi: 10.1145/2492007.2492017

Drachsler, H., y Kalz, M. (2016). The MOOC and learning analytics innovation cycle (MOLAC): a reflective summary of ongoing research and its challenges. Journal of Computer Assisted Learning 32(3), 281-290. doi: 10.1111/jcal.12135

Fini, A. (2009). The technological dimension of a massive open online course: The case of the CCKo 8 course tools. The International Review of Research in Open and Distance Learning, $10(5)$.

García Aretio, L. (2015). MOOC: ¿tsunami, revolución o moda pasajera? RIED. Revista Iberoamericana de Educación a Distancia, 18(1), 9-21. doi: 10.5944/ ried.18.1.13812

Gartner (2015). Hype Cycle for Education. Recuperado de https://www.gartner. com / doc/3090218/hype-cycleeducation-

Gartner (2016). Hype Cycle for Education, 2016. Recuperado de https://www. gartner.com/doc/3364119/hype-cycleeducation-

Iyer, G., y Katona, Z. (2015). Competing for Attention in Social Communication Markets. Social Science Research Network. doi: 10.2139/ssrn.2322435

Jordan, K. (2014). Initial Trends in Enrolment and Completion of Massive Open Online Courses. The International Review of Research in Open and Distance Learning, 15(1), 133-160.

Kop, R. (2011). The challenges to connectivist learning on open online networks: learning experiences during a Massive Open Online Course. The International Review of Research in Open and Distance Learning, 12(3), 19-38.

Kop, R., Fournier, H., y Mak, J. S. F. (2011). A pedagogy of abundance or a pedagogy to support human beings? Participant support on Massive Open Online Courses. The International Review of Research in
Open and Distance Learning, 12(7), 7493.

Martín, O., González, F., y García, M. A. (2013) Propuesta de evaluación de la calidad de los MOOCs a partir de la Guía Afortic. Revista Campus Virtuales, II(1), 124-132.

Méndez García, C. M. (2013). Diseño e implementación de cursos abiertos masivos en línea (MOOC): expectativas y consideraciones prácticas. RED. Revista de Educación a Distancia, 39.

Mengual-Andrés, S., Roig Vila, R., y Lloret Catalá, C. (2015). Validación del cuestionario de evaluación de la calidad de cursos virtuales adaptado a MOOC. RIED. Revista Iberoamericana de Educación a Distancia, 18(2), 145-169. doi: 10.5944/ ried.18.2.13664.

OnlineCourses.com. Top 10 Online Course Providers for 2016. Recuperado de http:// www.onlinecourses.com/

Poy, R., y Gonzales-Aguilar, A. (2014). Factores de éxito de los MOOC: algunas consideraciones críticas. Revista Ibérica de Sistemas y Tecnologías de la Información, E1, 105-118. doi: 10.4304/ risti.e1.105-118

Reutemann, J. (2016). Differences and Commonalities - A comparative report of video styles and course descriptions on edX, Coursera, Futurelearn and Iversity. En M. Khalil, M. Ebner, M. Kopp, A. Lorenz y M. Kalz, Proceedings of the European Stakeholder summit on experiences and best practices in and around MOOCs (EMOOCS 2016). (383392). Graz: Books on Demand.

Rodríguez-Ascaso, A., y Boticario, J. G. (2015). Accesibilidad y MOOC: Hacia una perspectiva integral. RIED. Revista Iberoamericana de Educación a Distancia, 18(2), 61-85. doi: 10.5944/ ried.18.2.13670

Torres Mancera, D., y Gago Saldaña, D. (2014). Los MOOC y su papel en la creación de comunidades de 
aprendizaje y participación. RIED. Revista Iberoamericana de Educación a Distancia, 17(1), 13-34. doi: 10.5944/ ried.17.1.11570

Valverde Berrocoso, J. (2014). MOOCs: una visión crítica desde las ciencias de la educación. Profesorado: Revista de curriculum y formación del profesorado, 18(1), 93-111.

Vázquez-Cano, E., y López Meneses, E. (2015). La filosofía educativa de los
MOOC y la educación universitaria. RIED. Revista Iberoamericana de Educación a Distancia, 18(2), 25-37. doi: 10.5944/ ried.18.2.14261

Zapata-Ros, M. (2013). MOOCs, una visión crítica y una alternativa complementaria: La individualización del aprendizaje y de la ayuda pedagógica. Revista Campus Virtuales, II(1), 20-38.

\section{PERFIL ACADÉMICO Y PROFESIONAL DE LOS AUTORES}

Ángel González de la Fuente. Licenciado en Periodismo por la Universidad Complutense de Madrid. Experto en gamificación para la educación, comunicación corporativa y comunicación audiovisual en la red. Responsable de proyectos e innovación en Telefónica Educación Digital. Responsable del portal MiríadaX en Telefónica. Docente y coordinador del MOOC de 'Introducción a la gamificación para docentes' del portal ScolarTIC.

E-mail: angel.gonzalezdelafuente@telefonica.com

\section{DIRECCIÓN DEL AUTOR}

Distrito Telefónica - Edificio Oeste 1, Planta $4^{\mathrm{a}}$

Ronda de la Comunicación s/n

Código Postal 28050

Madrid (España)

David Carabantes Alarcón. Profesor de la Universidad Complutense de Madrid (UCM), Doctor, Licenciado en Documentación, Máster en Nuevas Tecnologías Aplicadas a la Educación y Especialista Universitario en Gestión de la Investigación y de la Transferencia del Conocimiento. Coordinador del MOOC Gestión de la Información Científica en Abierto de MiríadaX. Miembro del grupo de investigación de la UCM "Políticas de información, tecnologías de la documentación y la comunicación científica (POLITECOM)".

E-mail: dcaraban@ucm.es

\section{DIRECCIÓN DEL AUTOR}

Universidad Complutense de Madrid Avda. Complutense, s/n 
Código Postal 28039

Madrid (España)

Fecha de recepción del artículo: 05/07/2016

Fecha de aceptación del artículo: 07/09/2016

\section{Como citar este artículo:}

González de la Fuente, A., y Carabantes Alarcón, D. (2017). MOOC: medición de satisfacción, fidelización, éxito y certificación de la educación digital. RIED. Revista Iberoamericana de Educación a Distancia, 20(1), pp. 105-123. doi: http://dx.doi. org/10.5944/ried.20.1.16820 